\title{
Genomic characterization of two Staphylococcus epidermidis bacteriophages with anti-biofilm potential
}

\author{
Diana Gutiérrez, Beatriz Martínez, Ana Rodríguez and Pilar García*
}

\begin{abstract}
Background: Staphylococcus epidermidis is a commensal bacterium but can colonize the hospital environment due to its ability to form biofilms favouring adhesion to host tissues, medical devices and increasing resistance to antibiotics. In this context, the use of phages to destroy biofilms is an interesting alternative.

Results: The complete genomes of two Staphylococcus epidermidis bacteriophages, vB_SepiS-philPLA5 and VB_SepiS-philPLA7, have been analyzed. Their genomes are 43,581 bp and 42,123 bp, and contain 67 and 59 orfs. Bioinformatic analyses enabled the assignment of putative functions to 36 and 29 gene products, respectively, including DNA packaging and morphogenetic proteins, lysis components, and proteins necessary for DNA recombination, regulation, modification and replication. A point mutation in VB_SepiS-philPLA5 lysogeny control-associated genes explained its strictly lytic behaviour. Comparative analysis of phi-IPLA5 and phi-IPLA7 genome structure resembled those of $\mathrm{S}$. epidermidis $\varphi \mathrm{PH} 15$ and $\varphi \mathrm{CNPH} 82$ phages. A mosaic structure of $S$. epidermidis prophage genomes was revealed by PCR analysis of three marker genes (integrase, major head protein and holin). Using these genes, high prevalence (73\%) of phage DNA in a representative S. epidermidis strain collection consisting of 60 isolates from women with mastitis and healthy women was determined. Putative pectin lyase-like domains detected in virion-associated proteins of both phages could be involved in exopolysaccharide (EPS) depolymerization, as evidenced by both the presence of a clear halo surrounding the phage lysis zone and the phage-mediated biofilm degradation.

Conclusions: Staphylococcus epidermidis bacteriophages, vB_SepiS-philPLA5 and VB_SepiS-philPLA7, have a mosaic structure similar to other widespread S. epidermidis prophages. Virions of these phages are provided of pectin lyase-like domains, which may be regarded as promising anti-biofilm tools.
\end{abstract}

\section{Background}

Staphylococcus epidermidis is a common skin and mucous commensal of healthy humans, and can easily be transmitted to medical devices being a serious clinical problem and one of the major causes of nosocomial infections [1] as well as mastitis in lactating women [2]. In the animal health context $S$. epidermidis has also been recognized as one of the main etiological agents of ovine and bovine mastitis [3].

S. epidermidis is a key factor in the transmission of virulence factors and it is involved in balancing epithelial

\footnotetext{
*Correspondence: pgarcia@ipla.csic.es

DairySafe Group, Department of Technology and Biotechnology of Dairy Products, Instituto de Productos Lácteos de Asturias (IPLA-CSIC), Paseo Río Linares, s/n, 33300, Villaviciosa, Asturias, Spain
}

microbiota. In contrast to $S$. aureus, S. epidermidis does not encode many virulence factors, but it can colonize the hospital environment due to its ability to form biofilms favouring adhesion to host tissues, medical devices and increasing resistance to antibiotics [4]. In addition, the enormous flexibility of this bacterium continuously generates continuously novel phenotypic and genotypic variants. Hospital isolates are often characterised by the carriage of several staphylococcal chromosome cassettes (SCCmec), conferring methicillin resistance [5]. Moreover, nosocomial S. epidermidis strains typically harbour multiple copies of the insertion sequence element IS256 in their genomes, which contribute to genetic adaptation during infection [6]. Recently, the first S. epidermidis pathogenicity

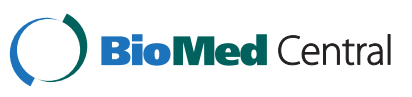


island (SePI), which encodes the staphylococcal enterotoxin SEC3 and SElL, has been described [7].

The widespread use of antibiotics in both humans and animals has led to the emergence of infectious bacteria resistant to a wide range of antimicrobials that greatly hinders their treatment. As a result of the search for complementary agents to antibiotics, phage therapy has resurfaced as means to prevent and treat infectious diseases. Phages have already been tested as anti-infectives in humans and animals [8], and phage-encoded lytic proteins may also be used to inhibit pathogenic bacteria [9]. In addition, the use of phages to destroy biofilms has gained much interest over the past years [10]. However, scarce information exists regarding the role of phages in eliminating $S$. epidermidis biofilms [11,12]. This is probably due to the limited number of phages infecting this species that have been characterized so far [13-15].

We have previously isolated and characterized three phages infecting S. epidermidis strains which belong to the Siphoviridae family (vB_SepiS-phiIPLA5, vB_SepiSphiIPLA6, and vB_SepiS-philPLA7) [15]. Phage vB_SepiSphiIPLA5 (hereafter phi-IPLA5) behaved as a virulent phage, probably derived from vB_SepiS-phiIPLA6, while vB_SepiS-phiIPLA7 (phi-IPLA7) was temperate. Both phages exhibited plaques surrounded by an increasing halo zone indicative of a polysaccharide depolymerase activity [16]. Moreover, in challenge assays phi-IPLA5 had lytic capability against $S$. epidermidis [15].

In the present work, the complete genome of phages phi-IPLA5 and phi-IPLA7 has been sequenced, annotated and compared with those previously described for staphylococcal phages. Genes encoding putative depolymerase activities were identified in these genomes. In addition, a representative $S$. epidermidis strain collection has been analyzed by using a multiplex PCR and the frequency of certain prophage groups determined. This study thus provides the basis for the evaluation of phages to control S. epidermidis strains.

\section{Results and discussion}

Due to the renewed interest in phage therapy and the ability of phages to successfully combat infections in both animals and humans, the aim of this work was the genetic characterization of two new $S$. epidermidis phages (phi-IPLA5 and phi-IPLA7) to investigate their potential as antimicrobials and, more specifically, as anti-biofilm agents based on our previous observations of the presence of an increasing halo surrounding the lysis plaques, indicating a depolymerase activity [15].

\section{Genome overview of phi-IPLA5 and phi-IPLA7 phages}

Both phages have a linear, double-stranded DNA genome consisting of 43,581 bp encoding 67 putative orfs in phi-IPLA5, while the phi-IPLA7 genome was 42,123 bp and 59 putative orfs were identified (Additional file 1: Table S1 and Additional file 2: Table S2; Figure $1 \mathrm{~A}$ and B). The $\mathrm{G}+\mathrm{C}$ content of phi-IPLA5 and phi-IPLA7 was $34.7 \%$, which is slightly higher than that of S. epidermidis strains (32\%) [17]. A BLASTN search revealed that nucleotide sequence of phi-IPLA5 and phi-IPLA7 shared a high degree of similarity with the other two S. epidermidis phages phiPH15 and phiCNPH82 (64\% and 65\%, for phiIPLA5, and $81 \%$ and $67 \%$ for phi-IPLA7, respectively) [14]. Bioinformatic analysis revealed a similar organization of the two phages in five functional modules (packaging, structure/morphogenesis, host lysis, lysogeny and replication/regulation) that perfectly fits the general structure of most double-stranded DNA bacteriophages [18]. Several putative promoters and terminators in phi-IPLA5 and in phi-IPLA7 were found by searching for the S. aureus $\sigma^{70}$ dependent promoter consensus motif (Figure 1A and B, Additional file 3: Table S3). The deduced promoter positions are consistent with a modular arrangement and imply that there is modular control of gene expression. The putative transcription pattern of both phages reveals two main groups of orfs running divergently and one of them includes the lysogenic cassette.

The amino acid (aa) sequences of the predicted orfs were searched for similarities to sequences from the available databases (Additional file 1: Table S1 and Additional file 2: Table S2). Significant matches were obtained for 36 orfs from phi-IPLA5 and 29 orfs from phi-IPLA7 and biological functions were assigned. No tRNA genes were found. No virulence genes were clearly identified.

In the DNA packaging module, the putative large terminase subunit of phi-IPLA5 and phi-IPLA7 showed homology (59\%) with those belonging to the pac-type phages such as Bacillus subtilis phage SPP1. It has been suggested that different functional classes of phageencoded terminases can be predicted from their amino acid sequence [19]. Confirmation of this result was obtained by restriction analysis of phi-IPLA5 and phiIPLA7 DNAs with the endonuclease XbaI. The phage genomes both have two cut sites but produced two single bands on agarose gels (data not shown), which suggests that both phi-IPLA5 and phi-IPLA7 genomes were circularly permuted.

In the structural module, the predicted major head and major tail proteins had a molecular mass consisting with previous protein analysis of virion particles, which showed a major polypeptide $(34 \mathrm{kDa})$ in phage phi-IPLA5 and two main proteins (27.5 and $34 \mathrm{kDa})$ in phi-IPLA7, respectively [15]. Putative virion-associate hydrolases (phiIPLA5 gp18 and phi-IPLA7 gp17) with an aminoterminal endopeptidase tail domain and a SGNH hydrolase domain related with lipases and esterases, were identified. Finally, pre neck appendage proteins (phi-IPLA5 gp19 and phiIPLA7 gp18) with pectin-lyase like domains weer identified 


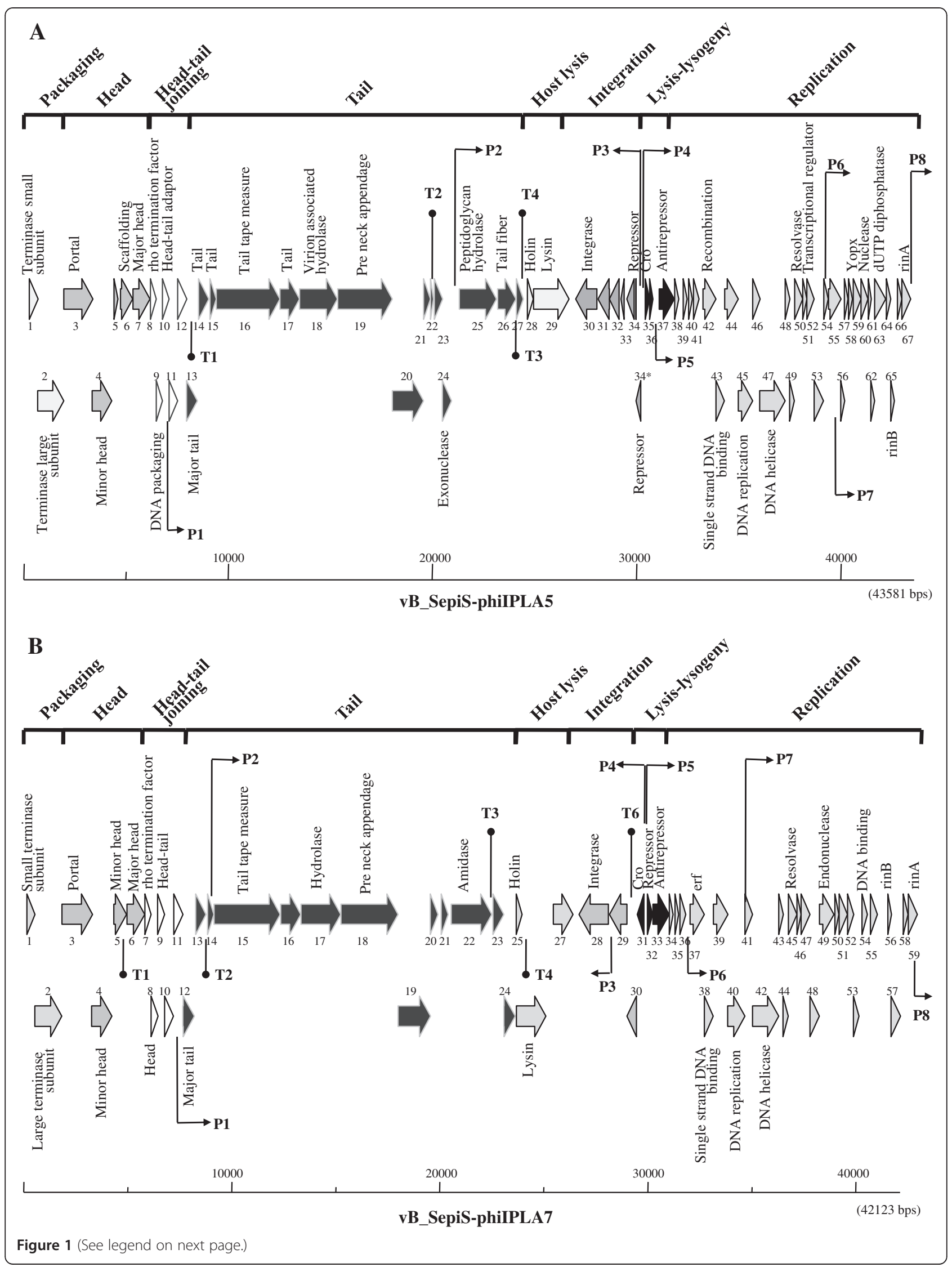


(See figure on previous page.)

Figure 1 Physical and genetic map of phages phi-IPLA5 (A) and phi-IPLA7 (B). The ORFs are sequentially numbered, indicated by arrows proportional to their lengths and pointing toward their direction of transcription. Some ORFs have been placed below for clarity. The functional modules are indicated on top of the scheme, and the names of several putatively or experimentally identified genes are shown. Putative promoter $(\mathrm{P})$ and terminator $(\mathrm{T})$ sequences are also indicated.

that could also be involved in the extracellular material degradation.

Phage phi-IPLA5, although strictly lytic, encoded a deficient lysis-lysogeny module. The phi-IPLA5 gp34 and phiIPLA5 gp34* proteins shared extended similarity with repressors of the $\mathrm{CI}$ type. A one-base replacement that shifted a TAC codon to the stop codon TAA was mapped at $29941 \mathrm{nt}$ resulting in a second $\operatorname{orf}\left(\operatorname{orf} 34^{*}\right)$. No RBS upstream of orf34\% could be detected. The presence of a truncated CI repressor in phi-IPLA5 would explain why the phage was unable to lysogenize [15]. In the cI-cro intergenic regions of both phages, two adjacent and outwardfacing putative promoters for cro and repressor genes were identified (Figure 1A and B, Additional file 3: Table S3). Additionally, two 7-bp direct repeats overlapping the two putative promoters in phi-IPLA7 were recognized (data not shown). These sequences might be putative operators for the binding of $\mathrm{CI}$ repressor which have been reported as regulators in the lysogeny module gene expression [20].

In the replication module, both phages contained DNA replication proteins (phi-IPLA5 gp45 and phiIPLA7 gp40) with DnaB domains which are essential in replication initiation, as well as DnaD domains which are a component of the primosome. phi-IPLA5 gp50 and phi-IPLA7 gp45 displayed homology to a Holliday junction resolvase (RusA) [21]. phi-IPLA5 gp59 had homology with Yopx proteins, an uncharacterized, wellconserved family of proteins found in bacteriophage and prophage regions of Gram-positive bacteria. A putative dUTPase gene gp63 was predicted in phi-IPLA5 genome which is highly conserved in several staphylococcal and lactococcal phages [22,23]. Gp65 and gp67 from phiIPLA5 and gp56 and gp59 from phi-IPLA7 displayed similarity to the RinA and RinB family of transcriptional regulators. Recently, the RinA family proteins have been showed as activators required for transcription of the late operon in temperate staphylococcal phages [24].

\section{Comparative genomics of phi-IPLA5 and phi-IPLA7 phages}

BLASTN database searches with the complete genome sequence of phi-IPLA5 and phi-IPLA7 revealed similarity at the nucleotide level with the two previously described $S$. epidermidis phages PH15 and CNPH82 and with S. aureus phages such as phiEW, phi29, phi37, phi52A and phi55. Comparison of these phages using Mauve software revealed gene synteny among the four S. epidermidis phages. Four homology blocks mostly matched the morphogenesis and integration, the lysis-lysogeny, replication, and regulation modules, (Figure 2). Extensive similarity in the head and tail morphogenesis modules was observed among the $S$. epidermidis phages genomes and other Staphylococcus phages (Figure 2; Additional file 1: Table S1 and Additional file 2: Table S2). In fact, S. epidermidis phages PH15 and CNPH82 have been shown to be highly similar to class II clade C of $S$. aureus phages [14]. An exception was observed in the putative exonuclease encoding gene (orf24) from phi-IPLA5, which was not found in phi-IPLA7 or in other staphylococcal phages. The phi-IPLA5 lysis region seemed to be rather unique, specifically in the holin encoding gene (orf28), to which no similarity was detected within other S. epidermidis phages. The holin phi-IPLA5 gp28 protein showed similarity (50\%) to the holin from S. aureus phage 29 while the holin phi-IPLA7 gp25 protein was identical to those in phages PH15 and CNPH82. Similarly, the phi-IPLA5 lysin encoding gene (orf29) did not have any matching gene in other phages. Moreover, the lysin phiIPLA5 gp29 protein belongs to the amidase_2 family while those of phi.IPLA7 (gp26) and the lysins from S. epidermidis phages $\mathrm{PH} 15$ and CNPH82 were highly similar (99\%) and belong to the amidase_3 family. A similar pattern was observed in the region surrounding the integrase gene. The putative integrase gene (orf30) of phi-IPLA5 differed from that in phi-IPLA7, and the corresponding protein (gp30) was 70\% similar to that of S. aureus phi96, while the integrase phi-IPLA7 protein (gp28) resembled that of $S$. epidermidis $\mathrm{PH} 15$ and $\mathrm{CNPH} 82$. The phage integrases from the newly isolated phages belonged to different families (Additional file 1: Table S1 and Additional file 2: Table S2).

Finally, no similarity with other phages was detected for the antirepressor encoding gene (orf33) found in the phiIPLA7 lysis-lysogeny region, Based on these data, S. epidermidis PH15 and CNPH82 and S. aureus phages phiEW, phi29, phi37, phi52A and phi55 are the closest relatives to phi-IPLA5 and phi-IPLA7. The extensive similarity between these phages is mainly in the morphogenetic region as previously reported on other Siphoviridae phages [25]. The observed homology in other regions or modules supports the modular theory of phage evolution [26].

\section{Incidence and typing of prophages in S. epidermidis strains} Previous studies have shown that prophages integrated in the bacterial chromosomes are the most widespread mobile genetic elements in $S$. aureus strains, which tipically 


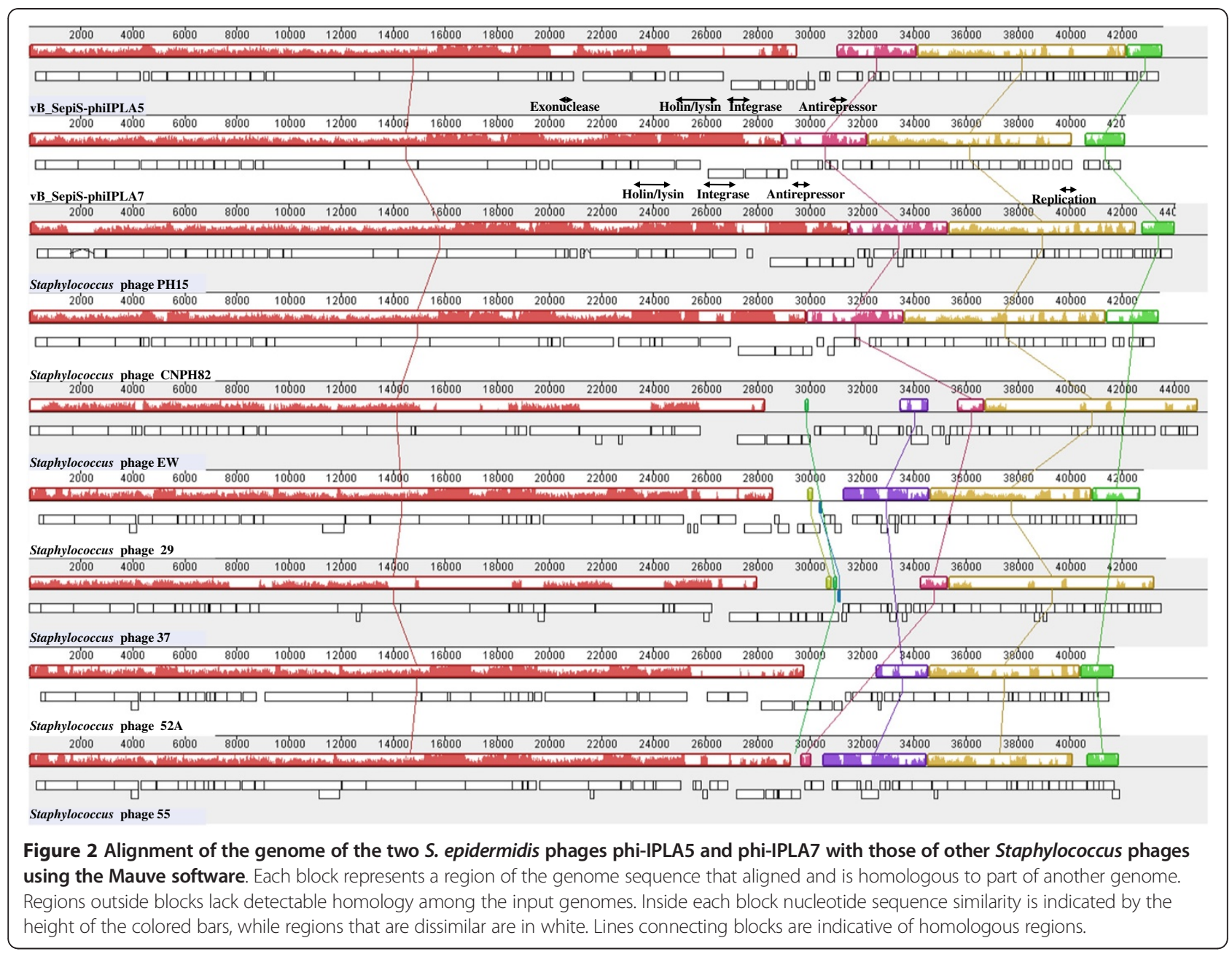

carry between one and four prophages [27]. However, the prophage content in $S$. epidermidis has not been determined to date. To approach this, the integrase (int), holin $(h o l)$ and major head protein $(m h p)$ genes were selected as marker modules as previously described in by [28] who studied genome mosaicism in prophages of $S$. aureus. Based on the genome wide comparison among phages and further in silico analysis of the integrase genes from the four S. epidermidis phages (phi-IPLA5, phi-IPLA7, PH15 and $\mathrm{CNPH} 82$ ), two groups were identified: int1 comprised by the highly similar int genes from phi-IPLA7, PH15 (95\%) and CNPH82 (99\%) and int2 composed by int phiIPLA5 to which no similarities were found among them. Likewise, S. epidermidis phage holin genes defined two groups: hol1 group comprised by hol phi-IPLA7, PH15 (91\%) and CNPH82 (100\%) and hol2 group that included hol phi-IPLA5. Finally, the phi-IPLA7 $m h p$ gene was very similar to phi-IPLA5 (97\%) and PH15 (95\%) but no counterpart was identified in CNPH82. Based on amino acid sequence homology this protein belongs to the phage- capsid superfamily. The presence or absence of this gene generated the mhp1 and mhp2 groups, respectively.

The presence of prophages in our S. epidermidis collection containing 60 isolates from women breast milk was investigated by a multiplex PCR to amplify the above mentioned genes (int, hol and $m h p$ ). Prophages of the group mhp1 were the most frequent (30\%) but none of the other targeted markers were amplified in these strains (Additional file 4: Table S4). About 10\% of the isolates were included in the mhp2 group and were mostly associated to the int 2 marker. We also observed that some phage groups were completely absent and others were less frequent. There were also some strains in which no amplification was observed pointing to the absence of prophages or the presence of other genes not detected by our PCR approach. It is remarkable that, besides the $10 \mathrm{~S}$. aureus bacteriophage integrase gene classes analysed previously [28], many other types of modules containing lysogenic functions, DNA replication, packaging, tail appendices and host lysis were 


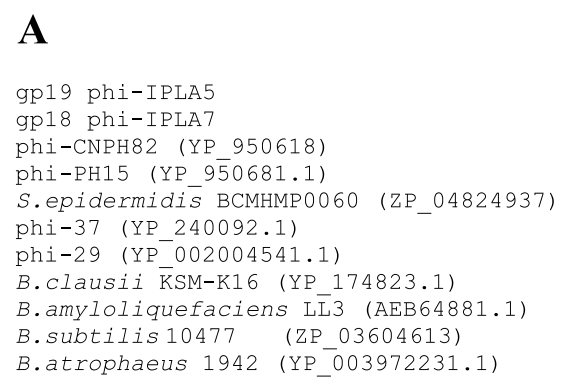

\section{B}

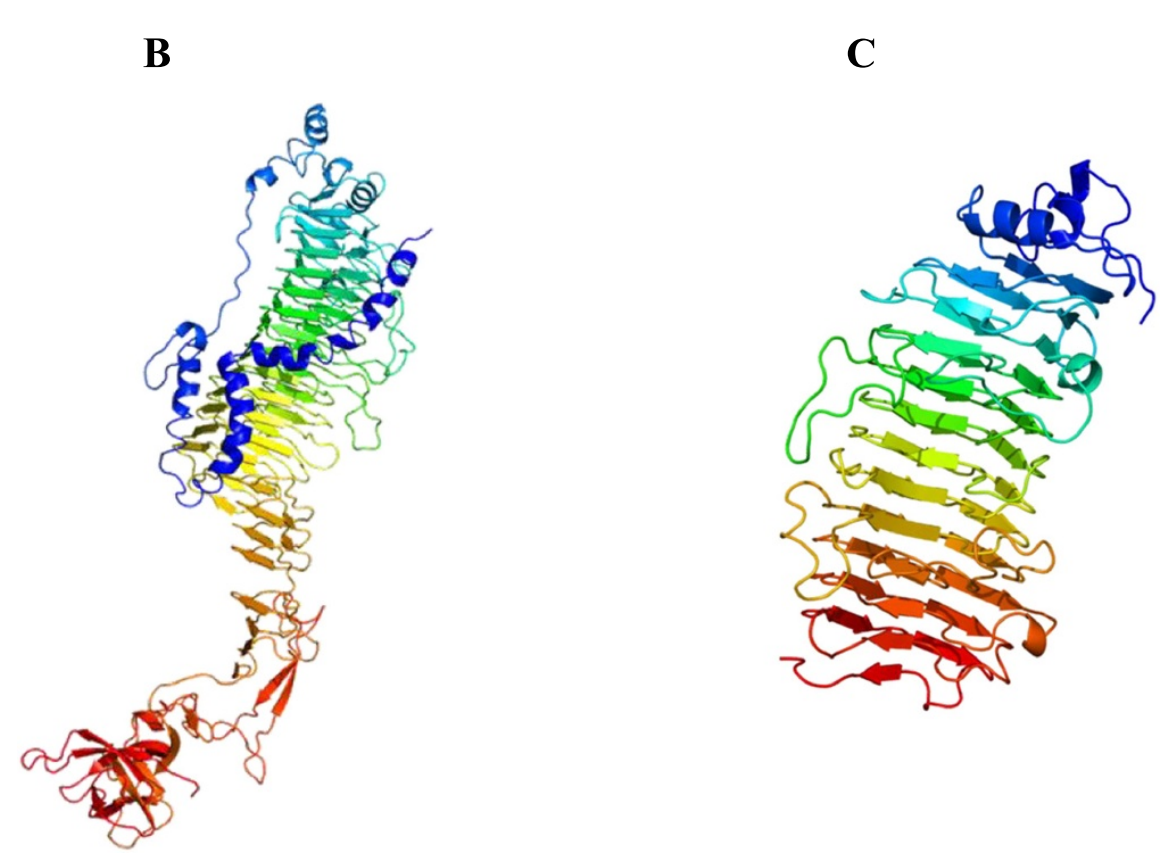

316 DDGITTHHSRYITISNCYAHTP------TGGSNNNGIEIDDGSQYVFLSNNRTKGNFGGLEIKAH 374 316 DDGITTHHSRYITISNCYAHTP------TGGSNNNGVEIDDGSQYVFLSNNRTKGNFGGLEIKAH 374 316 DDGITTHHSRYITISNCYAHTP------TGGSNNNGVEIDDGSOYVFLSNNRTKGNFGGLEIKAH 374 316 DDGITTHHSRYITISNCYAHDP------TGGSNSNGIEIDDGSQFVFLSNNRTKGNFGGLEIKAH 374 316 DDGITTHHSRYITISNCYAHTP------TGGSNNNGIEIDDGSQYVFLSNNRTKGNFGGLEIKAH 374 317 DDGITVHWSRYILITDCYSHDP------IGGGNNNGIEVDDGSQFVFLSDNKTENNYGGLEIKAH 375 198 DDGITTHHSOYINILNCYSHDP------RLTANCNGFEIDDGSRHVVLSNNRSKGCYGGIEIKAH 256 294 DDGFTTHHSNYLFIEGCFSTGATN-YLDIGHKNSNGFEMDDGSRFVFVSRCVSKENTRGYEVKGH 357 269 DDGITTHYSEYIFISNCHCTNPTGLAHAAGKANSNGIEIDDGSKHVWLLNNYTEGNIRGVEVKAH 333 253 DDGITTHYSEYIFISNCHSGNPRGTEFAEGVSNSNGIEIDDGSRHVWLLNNFTSGNIRGVEVKAH 317 253 DDGITTHYSEYIFISNCHSGNPRGTEHAEGISNSNGIEIDDGSKHVWLLNNFTSGNIRGVEVKAH 317

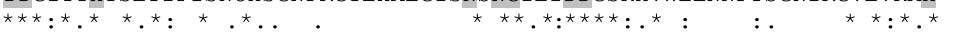

Figure 3 Aminoacid sequence analysis of the virion-associated protein gp18 from phi-IPLA7. A) ClustalW alignment of the predicted binding site. Positions with a single, fully conserved residue are marked with an asterisk; the colon marks the conserved residues between groups of strongly similar properties, and the period marks the residues weakly conserved between groups based on the Gonnet PAM 250 matrix, score $<0,5$. Highlighted are those residues involved in ligand association. B) Predicted 3D structure of phi-IPLA7 gp18. C) Predicted 3D structure of the domain pectin lyase like (D1) (amino acids 316-374).

described in S. aureus phages, revealing the high diversity and the mosaic structure of prophages in this species [22].

Previous studies suggested that the integrase type group in $S$. aureus strains is closely linked to the virulence gene content of prophages and might therefore convey information about their pathogenic potential [28]. We found that the largest group of $S$. epidermidis prophages belongs to int2 group, which was mostly found in mastitic strains. On the other hand, prevalence of prophages, as defined by the positive amplification of at least one marker gene, was higher ( $82 \%$ vs $59 \%)$ in strains producing mastitis. This result would support the hypothesis that prophages are directly involved in virulence [29]. However, no virulence factors have been described to date in S. epidermidis phages.

In our previous work, the yield of mitomycin $C$ inducible prophages in S. epidermidis was rather low (3\%) and we hypothesized that it could be due to the lack of appropriately sensitive host strains to detect them [15]. In view of the new PCR results, phage DNA sequences were present in $73 \%$ of the analyzed S. epidermidis strains. Although amplification of at least one of the markers does not necessarily mean that the bacterial strains are lysogens (i.e. marker genes in defective prophages), our results support the notion that lysogeny in S. epidermidis may be higher than anticipated and likely more frequent among clinical strains, as noted previously in S. aureus strains isolated from diverse clinical samples [28].

\section{Virion proteins with a putative hydrolytic activity of extracellular components}

A pectin lyase-like domain (aminoacids 117 to 539) was identified in the pre-neck appendage protein of both phages (Additional file 1: Table S1 and Additional file 2: Table S2). 
Recently, it has been demonstrated that some tail spike proteins similar to pectin lyases have exopolysaccharide (EPS) degrading activity [30]. Similar proteins to phi-IPLA5 gp19 and phi-IPLA7 gp18 were identified by BLASTp search in PH15 and CNPH82 phages, S. aureus phage 37, Bacillus phage phi29 and prophages in several Bacillus species (Figure 3A). Surprisingly, most of the phi-IPLA5 gp19 and phi-IPLA7 gp18 related proteins belonged to Bacillus sp and not to other staphylococcal species. It is remarkable that all the proteins analyzed were highly conserved at the predicted binding site (amino acids 316-374) and the residues responsible for the interaction with the ligand were shared by the different phages (Figure 3A). To support the predicted catalytic domains, the software Phyre ${ }^{2}$ was used to predict the tridimensional structure (3day) of the protein phi-IPLA7 gp18 to which, the primary structure of the counterpart protein from phi-IPLA5 was $98 \%$ identical. To model phi-IPLA7 gp18 (Figure 3B), the crystal structure of the bacteriophage phi29 gp12 neck protein from Bacillus subtilis [31] was used. $82 \%$ of phi-IPLA7 gp18 amino acids were predicted at a confidence level of $90 \%$. The pectin lyase-like domain (D1) (Figure 3C) displayed a righthanded continuous twelve helix repetition, where each coil of the helix featured three beta-strands and three turn regions. Proteins containing these repeats most often are enzymes with polysaccharide substrates, and it was demonstrated that this topology is shared by several proteins, with the variation in the number of coils, including bacterial pectate lyases, fungal and bacterial galacturonases and phage tail spikes [32]. On the other hand, no amino acid sequence homology was detected between phi-IPLA7 gp18 and Pseudomonas putida $\phi 15$ tail spike protein, although this also showed right handed beta helical folds identified in carbohydrate depolymerizing enzymes [30].

\section{Biofilm degradation by phi-IPLA5 and phi-IPLA7 phages}

Three lines of evidence were pointing to a putative antibiofilm activity by the newly isolated $S$. epidermidis phages phi-IPLA5 and phi-IPLA7. First, as previously reported [15], a halo surrounding the clear lysis plaques could be observed which increased with time. These halos are regarded as an indicator for the presence of phage-associated EPS depolymerases [16,30]. Secondly, a clear zone was previously observed when phages phiIPLA5 and phi-IPLA7 were dropped on some bacterial strains (drop-sensitive strains). These strains were resistant to infection meaning that no plaques were observed when the phage stock was diluted and plated on these strains [15]. Third, based on sequence comparison and $3 \mathrm{D}$ predictions, two candidate proteins were identified as putative EPS degrading enzymes. To investigate if these phages were involved in biofilm degradation, cell counts and phage titre of the clear zone where the phage suspension is dropped, and into the host cell lawn were carried out. As expected, lower bacterial counts were found when the phages were dropped onto the sensitive strain $S$. epidermidis F12 while viable counts of the resistant $S$. epidermidis CJBP3 remained unaltered (Figure 4). Interestingly, the clear zone generated on the drop-sensitive strains revealed similar bacterial counts to the bacterial
$\mathbf{A}$

I

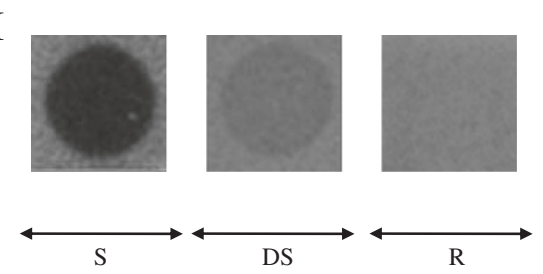

II

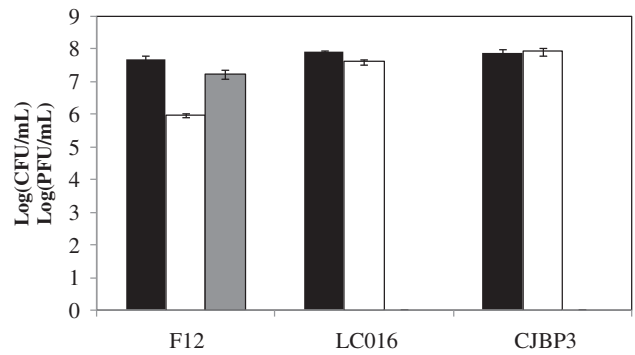

B

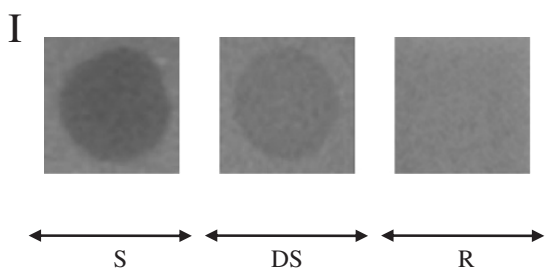

II

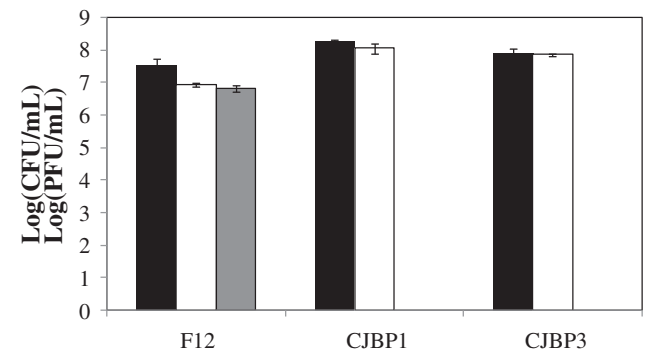

Figure 4 Effect of phages A) phi-IPLA5 and B) phi-IPLA7 on a lawn of S. epidermidis. I) Morphology of phage lytic zone when dropped on different S. epidermidis strains. (S) Sensitive strain; (R) resistant strain; (DS) drop-sensitive strain. II) Bacteria viable number in drop-zone (white), bacteria lawn (black) and phage titre (grey) of phages. Each value correspond with the mean of five different experiments, the standard error is represented by bars. 
lawn despite its distinct visual appearance. Moreover, $S$. epidermidis F12 clearly supported phage propagation, while no phages were detected when sampling the drop zone of the resistant and drop sensitive strains (Figure 4). Overall, these results supports the presence of phage depolymerase activity, although we can not totally discard our previous hypothesis that ascribed the clear zone on drop-sensitive strains to "lysis from without" [15].

In order to confirm the ability of the phages to mediate biofilm degradation, the three types of strains were grown in microtiter plates for 7 days for biofilm formation and subsequently challenged with phage phi-IPLA7. Viability of the sensitive S. epidermidis F12 in both planktonic and biofilm cells was readily reduced even after $1 \mathrm{~h}$ in the presence of the phage, and only $5 \%$ of the total counts survived (ANOVA; $\mathrm{p}<0.05$ ) after $3 \mathrm{~h}$ (Figure 5). In the case of the drop-sensitive S. epidermidis CJBP1, total cell numbers remained unchanged (ANOVA; $\mathrm{p}>0.05$ ) at both $1 \mathrm{~h}$ and $3 \mathrm{~h}$. However, a dramatic shift towards the planktonic state was observed in the presence of the phage and, after $3 \mathrm{~h}$, and just $2 \%$ of the cells remained attached (Figure 5B). These data support the ability of phage phi-IPLA7 to degrade the extracellular material of the biofilm, releasing the attached cells. On the contrary, neither the viability nor the biofilm formed by the resistant strain S. epidermidis CJBP3 was affected by phage phi-IPLA7 (ANOVA; $p>0.05$ ). A different composition in the extracellular biofilm material could explain this result. Studies on staphylococcal biofilm development suggest that the extracellular matrix consists of proteins, DNA, and/or polysaccharide (PIA) [1]. Recently, it has become evident that some strains are not reliant on PIA for biofilm formation [33].

\section{Conclusions}

In this work, we have presented a detailed genomic and molecular characterization of two new S. epidermidis phages, phi-IPLA5 and phi-IPLA7. Based on this, a multiplex PCR was designed that revealed a high prevalence of prophage and/or defective prophages within $S$. epidermidis strains. Genome mining detected the presence of virion-associated proteins with a putative EPS depolymerase activity. To our knowledge, this is the first time that degradation of extracellular material has been ascribed to $S$. epidermidis phages. Further studies to confirm the role of these proteins in phage-induced biofim destructuring are in progress with the hope to set the foundation of new anti-biofilm strategies.

\section{Methods}

\section{Bacterial growth conditions and phages propagation}

S. epidermidis F12 was used as the host strain for phages phi-IPLA5 and phi-IPLA7 [15]. A total of sixty S. epidermidis strains isolated from women's breast milk [2] were used in lysogeny analysis (Additional file 5: Figure S1). Staphylococcal cells were routinely cultured in TSB broth (Triptona Soy Broth, Scharlau, Barcelona, Spain) at $37^{\circ} \mathrm{C}$ with shaking or in TSB plates containing $2 \%(\mathrm{w} / \mathrm{v})$ bacteriological agar (TSA). Bacteriophages phi-IPLA5 and phi-IPLA7 were propagated as described previously [15].

\section{Phage genome sequencing and analysis}

To prepare bacterial DNA-free samples for sequence analysis, the purified phages were treated with DNaseI bovine pancreas (Sigma, Madrid, Spain) and the phage DNA was extracted as previously described [34]. The genome sequence of phages phi-IPLA5 and phi-IPLA7 was generated by ultra-high throughput GS FLX sequencing with 20-fold redundancy on average. Orfs were predicted with Clone Manager 7 version 7.10 software in all reading frames with a threshold of 40 codons. BLASTX and BLASTP (http://www.ncbi.nlm.nih.gov/blast/Blast. cgi) were used to search for homologous proteins. Structural predictions and motif searches were performed with InterProScan (http://www.ebi.ac.uk/InterProScan/). The search for putative tRNA encoding genes was
A

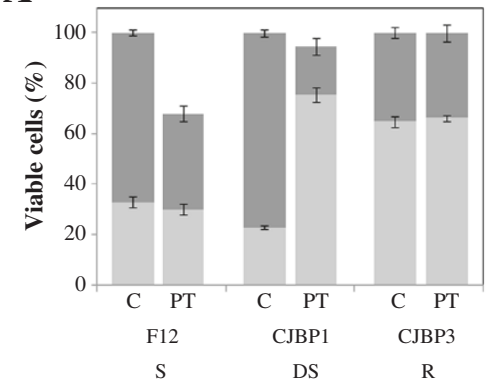

B

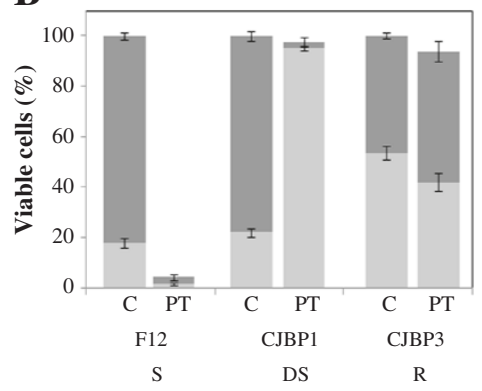

Figure 5 Killing of S. epidermidis cells forming a biofilm on microtiter wells by phage phi-IPLA7 after incubation for $1 \mathrm{~h}$ (A) and $3 \mathrm{~h}$ (B). Results are depicted as the percentage of attached cells (dark gray square) and planktonic cells (light gray square) detected in control biofilms treated with SM buffer (C) and in biofilms treated with phage phi-IPLA7 (PT). Each value corresponds with the mean of five different experiments and the standard error is represented by bars. 
performed with tRNAscan-SE 1.21 (http://selab.janelia. org/tRNAscan-SE/). $\sigma^{70}$ promoter sequences were identified using PPP (Prokaryotic Promoter Prediction at http:// bioinformatics.biol.rug.nl/websoftware/ppp/ppp_start.php). The rho-independent terminators were identified using the Trans Term program (http://nbc3.biologie.uni-kl.de) and energy was calculated by the mfold web server (http:// mfold.rna.albany.edu). Genomic comparisons at the nucleotide level were made with Mauve software, using a progressive alignment with default settings (http://gel. ahabs.wisc.edu/mauve/). 3 day structure prediction of proteins was made by using the bioinformatic software Phyre ${ }^{2}$ (http://gel.ahabs.wisc.edu/mauve/download.php). To predict the binding site of the ligands, the software 3Dligandsite was used (http://www.sbg.bio.ic.ac.uk/3dligandsite/).

\section{Accession numbers}

The sequences of phi-IPLA5 and phi-IPLA7 have been deposited in the GenBank under accession numbers JN192400 and JN192401, respectively.

\section{Lysogeny and prophage typing}

The presence of resident prophages was screened by multiplex PCR using the primers specified in Additional file 5: Figure S1. Total DNA extraction was carried out from $S$. epidermidis strains by using "GenElute ${ }^{\mathrm{TM}}$ Bacterial Genomic DNA Kit” (Sigma-Aldrich, Madrid, Spain). PCR reactions were performed using the kit 'PureTaq Ready-To$G^{\mathrm{TM}}$ PCR Beads' (GE Healthcare, Munich, Germany). As positive control, pure phage DNA from phi-IPLA5 and phi-IPLA7 was used. Gel images were processed using the software Quantity One software (BioRad Laboratories, Hercules, CA). The similarity matrix was calculated on the basis of the simple matching coefficient, and its corresponding dendrogram was deduced using the unweighted pair group method with arithmetic averages.

\section{Microbiological analysis of phage lysis zones and biofilms} To visualize the lysis area formation, $5 \mu \mathrm{l}$ of a $10^{8} \mathrm{PFU} /$ $\mathrm{ml}$ phage suspension was dropped on a $S$. epidermidis bacterial lawn and incubated at $37^{\circ} \mathrm{C}$. For comparison between the lysis zone and the bacterium lawn zone, an area with the same volume was removed from each zone, suspended in $200 \mu \mathrm{l}$ of SM buffer $(20 \mathrm{mM}$ Tris$\mathrm{HCl}, 10 \mathrm{mM} \mathrm{MgSO}_{4}, 10 \mathrm{mM} \mathrm{CaCl}, 100 \mathrm{mM} \mathrm{NaCl}, \mathrm{pH}$ $7.5)$ and vigorously vortexed. Phage titre was determined using the double layer agar method, while bacterial count was determined by plating serial dilutions on TSB agar. To determine the potential of phages to degrade biofilms, $S$. epidermidis $\mathrm{o} / \mathrm{n}$ cultures were diluted in fresh TSB to $10^{6} \mathrm{CFU} / \mathrm{ml}$, poured into a 96 microwell plate (Thermo Scientific, Madrid, Spain) and incubated during 7 day at $37^{\circ} \mathrm{C}$. Wells were washed twice with SM buffer and either $220 \mu \mathrm{l}$ of a phage stock $\left(10^{8} \mathrm{PFU} / \mathrm{ml}\right)$ or $220 \mu \mathrm{l}$ of SM buffer were added for test and control purposes, respectively. Plates were incubated for $1 \mathrm{~h}$ and $3 \mathrm{~h}$ and then supernatants and adhered cells were collected and plated for bacteria counting. The results were represented as the viable cells percentage respect the total cell number in the control wells without phage treatment (cells in the supernatant + cells adhered to the well).

Statistical analysis was performed by one-way analysis of variance (ANOVA) followed by the Bonferroni multicomparison test. Statistical significance was considered at $\mathrm{p}<0.05$.

\section{Additional files}

Additional file 1: Table S1. Features of bacteriophage phi-IPLA5 orfs, gene products (gp) and functional assignments.

Additional file 2: Table S2. Features of bacteriophage phi-IPLA7 orfs, gene products (gp) and functional assignments.

Additional file 3: Table S3. Putative promoters and terminators sequences of phi-IPLA5 and phi-IPLA7. -10 and -35 boxes are underlined. Nucleotide positions and presence of the TG dinucleotide were also indicated. At terminator sequences nucleotides in the stem-loop structure are underlined.

Additional file 4: Table S4. Primers used for multiplex PCR reactions.

Additional file 5: Figure S1. Multiplex PCR detecting the integrase, holin and major head protein genes in S. epidermidis strains. $(H)$ Strains isolated from healthy woman, (M) strains isolated from mastitic women. Absence and presence of a specific gene is represented by white and grey boxes, respectively.

\section{Competing interests}

The authors declare that they have no competing interests.

\section{Authors' contributions}

DG and PG performed bioinformatics analyses of nucleotide and protein sequences. PG, BM and AR designed the study, obtained funding and wrote the manuscript. All authors read and approved the final manuscript.

\section{Acknowledgments}

This research study was supported by grants AGL2009-13144-C02-01 (Ministry of Science and Innovation, Spain) and PIE200970I090 (CSIC, Spain) We acknowledge support of the publication fee by the CSIC Open Access Publication Support Initiative through its Unit of Information Resources for Research (URICI). D. G. is a fellow of the Ministry of Science and Innovation, Spain. We would like to thank R. Calvo (IPLA-CSIC) for her technical assistance. We also thank C. Billington (ESR, New Zealand) for critical reading of the manuscript and helpful discussions.

Received: 21 November 2011 Accepted: 17 May 2012 Published: 8 June 2012

\section{References}

1. Otto M: Staphylococcus epidermidis the "accidental" pathogen. Nat Rev 2009, 7:555-567

2. Delgado $S$, Arroyo R, Jiménez E, Marín ML, Del Campo R, Fernández L, Rodríguez JM: Staphylococcus epidermidis strains isolated from breast milk of women suffering infectious mastitis: potential virulence traits and resistance to antibiotics. BMC Microbio/ 2009, 9:82.

3. Oliveira M, Nunes SF, Carneiro C, Bexiga R, Bernardo F, Vilela CL: Time course of biofilm formation by Staphylococcus aureus and Staphylococcus epidermidis mastitis isolates. Vet Microbiol 2007, 124:187-191.

4. Jabbouri S, Sadovskaya I: Characteristics of the biofilm matrix and its role as a possible target for the detection and eradication of Staphylococcus epidermidis associated with medical implant infections. FEMS Immunol Med Microbiol 2010, 59:280-291. 
5. Schoenfelder SM, Lange C, Eckart M, Hennig S, Kozytska S, Ziebuhr W: Success through diversity - how Staphylococcus epidermidis establishes as a nosocomial pathogen. Int J Med Microbiol 2010, 300:380-386.

6. Kozitskaya S, Cho SH, Dietrich K, Marre R, Naber K, Ziebuhr W: The bacterial insertion sequence element IS256 occurs preferentially in nosocomial Staphylococcus epidermidis isolates: association with biofilm formation and resistance to aminoglycosides. Infect Immun 2004, 72:1210-1215.

7. Madhusoodanan J, Seo KS, Remortel B, Park JY, Hwang SY, Fox LK, Park YH, Deobald CF, Wang D, Liu S, Daugherty SC, Gill AL, Bohach GA, Gill SR: An enterotoxin-bearing pathogenicity island in Staphylococcus epidermidis. J Bacteriol 2011, 193:1854-1862.

8. Kutateladze M, Adamia R: Bacteriophages as potential new therapeutics to replace or supplement antibiotics. Trends Biotechnol 2010, 28:591-595.

9. Fischetti VA: Bacteriophage endolysins: a novel anti-infective to control Gram-positive pathogens. Int J Med Microbiol 2010, 300:357-362.

10. Donlan RM: Preventing biofilms of clinically relevant organisms using bacteriophage. Trends Microbiol 2009, 17:66-72.

11. Cerca N, Oliveira R, Azeredo J: Susceptibility of Staphylococcus epidermidis planktonic cells and biofilms to the lytic action of Staphylococcus bacteriophage K. Lett Appl Microbiol 2007, 45:313-317.

12. Curtin JJ, Donlan RM: Using bacteriophages to reduce formation of catheter-associated biofilms by Staphylococcus epidermidis. Antimicrob Agents Chemother 2006, 50:1268-1275.

13. Aswani V, Tremblay DM, Moineau S, Shukla SK: Staphylococcus epidermidis bacteriophages from the anterior nares of humans. Appl Environ Microbiol 2011, 77:7853-7855.

14. Daniel A, Bonnen PE, Fischetti VA: First complete genome sequence of two Staphylococcus epidermidis bacteriophages. J Bacterio/ 2007 189:2086-2100.

15. Gutiérrez D, Martínez B, Rodríguez A, García P: Isolation and characterization of bacteriophages infecting Staphylococcus epidermidis. Curr Microbiol 2010, 61:601-608.

16. Hughes KA, Sutherland IW, Jones MV: Biofilm susceptibility to bacteriophage attack: the role of phage-borne polysaccharide depolymerase. Microbiology 1998, 144:3039-3047.

17. Cates S: NCBI: National Center for Biotechnology Information. 2006. http://cnx.org/content/m11789/1.3/

18. Brüssow $H$, Desiere F: Comparative phage genomics and the evolution of Siphoviridae: insights from dairy phages. Mol Microbiol 2001, 39:213-222.

19. Casjens SR, Gilcrease EB, Winn-Stapley DA, Schicklmaier P, Schmieger H, Pedulla ML, Ford ME, Houtz JM, Hatfull GF, Hendrix RW: The generalized transducing Salmonella bacteriophage ES18: complete genome sequence and DNA packaging strategy. J Bacteriol 2005, 187:1091-1104.

20. García P, Ladero V, Alonso JC, Suárez JE: Cooperative interaction of Cl protein regulates lysogeny of Lactobacillus casei by bacteriophage A2. J Virol 1999, 73:3920-3929.

21. Mahdi AA, Sharples GJ, Mandal TN, Lloyd RG: Holliday junction resolvases encoded by homologous rusA genes in Escherichia coli K-12 and phage 82. J Mol Biol 1996, 257:561-573.

22. Kahánková J, Pantůček R, Goerke C, Rưžičková V, Holochová P, Doškař J: Multilocus PCR typing strategy for differentiation of Staphylococcus aureus siphoviruses reflecting their modular genome structure. Environ Microbiol 2010, 12:2527-2538.

23. Labrie S, Moineau S: Complete genomic sequence of bacteriophage ul36: demonstration of phage heterogeneity within the P335 quasi-species of lactococcal phages. Virol 2002, 296:308-320.

24. Ferrer MD, Quiles-Puchalt N, Harwich MD, Tormo-Más MA, Campoy S, Barbé J, Lasa I, Novick RP, Christie GE, Penadés JR: RinA controls phage-mediated packaging and transfer of virulence genes in Gram-positive bacteria. Nucleic Acids Res 2011, 39:5866-5878.

25. Proux C, van Sinderen D, Suarez J, García P, Ladero V, Fitzgerald GF, Desiere F, Brüssow $\mathrm{H}$ : The dilemma of phage taxonomy illustrated by comparative genomics of Sfi21-like Siphoviridae in lactic acid bacteria. J Bacterio/ 2002, 184:6026-6036.

26. Brüssow H, Canchaya C, Hardt WD: Phages and the evolution of bacterial pathogens: from genomic rearrangements to lysogenic conversion. Microbiol Mol Biol Rev 2004, 68:560-602.

27. Pantůček R, Doskar J, Růzicková V, Kaspárek P, Orácová E, Kvardová V, Rosypal S: Identification of bacteriophage types and their carriage in Staphylococcus aureus. Arch Virol 2004, 149:1689-1703.
28. Goerke C, Pantůček R, Holtfreter S, Schulte B, Zink M, Grumann D, Bröker BM, Doskar J, Wolz C: Diversity of prophages in dominant Staphylococcus aureus clonal lineages. J Bacterio/ 2009, 191:3462-3468.

29. Boyd EF, Brüssow H: Common themes among bacteriophage-encoded virulence factors and diversity among the bacteriophages involved. Trends Microbiol 2002, 10:521-529.

30. Cornelissen A, Ceyssens PJ, T'Syen J, Van Praet H, Noben JP, Shaburova OV, Krylov VN, Volckaert G, Lavigne R: The T7-related Pseudomonas putida phage $\varphi 15$ displays virion-associated biofilm degradation properties. PLoS One 2011, 6:e18597.

31. Xiang Y, Leiman PG, Li L, Grimes S, Anderson DL, Rossmann MG Crystallographic insights into the autocatalytic assembly mechanism of a bacteriophage tail spike. Mol Cell 2009, 34:375-386.

32. Jenkins J, Mayans O, Pickersgill R: Structure and evolution of parallel beta-helix proteins. J Struct Biol 1998, 122:236-246.

33. Tormo MA, Knecht E, Götz F, Lasa I, Penadés JR: Bap-dependent biofilm formation by pathogenic species of Staphylococcus: evidence of horizontal gene transfer? Microbiology 2005, 151:2465-2475.

34. Sambrook J, Fritsch EF, Maniatis T: Molecular cloning: a laboratory manual. 2nd edition. Cold Spring Harbor, NY, Cold Spring Harbor Laboratory; 1989.

doi:10.1186/1471-2164-13-228

Cite this article as: Gutiérrez et al:: Genomic characterization of two Staphylococcus epidermidis bacteriophages with anti-biofilm potential. BMC Genomics 2012 13:228.

\section{Submit your next manuscript to BioMed Central and take full advantage of:}

- Convenient online submission

- Thorough peer review

- No space constraints or color figure charges

- Immediate publication on acceptance

- Inclusion in PubMed, CAS, Scopus and Google Scholar

- Research which is freely available for redistribution 